\title{
Linguistik und Medizin
}




\section{Sprache und Wissen}

Herausgegeben von Ekkehard Felder

Wissenschaftlicher Beirat

Markus Hundt, Wolf-Andreas Liebert, Thomas Spranz-Fogasy, Berbeli Wanning, Ingo H. Warnke und Martin Wengeler

\section{Band 44}




\section{Linguistik und Medizin}

Sprachwissenschaftliche Zugänge und interdisziplinäre Perspektiven

Herausgegeben von

Marina lakushevich, Yvonne Ilg

und Theresa Schnedermann 
Gefördert durch die Deutsche Forschungsgemeinschaft (DFG) - IA 78/1-1

ISBN 978-3-11-068864-1

e-ISBN (PDF) 978-3-11-068869-6

e-ISBN (EPUB) 978-3-11-068878-8

ISSN 1864-2284

DOI https://doi.org/10.1515/9783110688696

\section{(cc) BY-NC-ND}

Dieses Werk ist lizenziert unter einer Creative Commons Namensnennung - Nicht-kommerziell Keine Bearbeitung 4.0 International Lizenz. Weitere Informationen finden Sie unter http://creativecommons.org/licenses/by-nc-nd/4.0/.

\section{Library of Congress Control Number: 2021945811}

\section{Bibliografische Information der Deutschen Nationalbibliothek}

Die Deutsche Nationalbibliothek verzeichnet diese Publikation in der Deutschen Nationalbibliografie; detaillierte bibliografische Daten sind im Internet über http://dnb.dnb.de abrufbar.

(C) 2021 bei den Autorinnen und Autoren, Zusammenstellung (c) 2021 Marina lakushevich, Yvonne Ilg und Theresa Schnedermann, publiziert von Walter de Gruyter GmbH, Berlin/Boston Dieses Buch ist als Open-Access-Publikation verfügbar über www.degruyter.com.

Satz: Integra Software Services Pvt. Ltd.

Druck und Bindung: $\mathrm{CPI}$ books $\mathrm{GmbH}$, Leck

www.degruyter.com 